\title{
Auditoria clínica do tratamento primário de fraturas expostas: Tratamento antimicrobiano e profilaxia antitetânica*
}

\section{Clinical Audit of Primary Treatment of Open Fractures: Antibiotic Treatment and Tetanus Prophylaxis}

\author{
Adriana Lúcia Ferris de Assunção ${ }^{10}$ Sílvia Teodoro de Oliveira ${ }^{10}$ \\ ${ }^{1}$ Fundação Hospitalar de Minas Gerais, Hospital João XXIII, Belo \\ Endereço para correspondência Adriana Lúcia Ferris de Assunção, \\ Horizonte, MG, Brasil \\ Rua João Donada, 453, Bairro Santa Terezinha, Belo Horizonte, MG, \\ CEP: 31.360-190, Brasil (e-mail: adriana.ferris@hotmail.com).
}

Rev Bras Ortop 2020;55(3):284-292.

\section{Resumo \\ Palavras-chave \\ - fraturas expostas \\ - antibioticoprofilaxia \\ - infecção \\ - tétano}

Objetivo Avaliar se as condutas envolvendo o tratamento antimicrobiano e a profilaxia contra o tétano têm sido realizadas conforme o Protocolo Clínico da Instituição.

Métodos Estudo descritivo e retrospectivo, realizado em pacientes de ambos os gêneros, $>18$ anos, admitidos em um hospital público estadual especializado em urgência e trauma, para tratamento primário de fratura exposta. Os dados de interesse foram pesquisados em prontuários médicos, prescrições de medicamentos, relatórios de pacientes admitidos no Bloco Cirúrgico e solicitações de profilaxia antitetânica. Resultados Foram selecionados 241 pacientes, a maioria homens $(81,7 \%)$, adultos jovens (64,3\%), vítimas de acidentes motociclísticos (53,5\%). As complicações infecciosas estiveram presentes em $18,7 \%$ das fraturas, o tempo médio para a abordagem cirúrgica foi de 4 horas e 12 minutos, e $91,7 \%$ dos pacientes tiveram prescrição do tratamento antimicrobiano no pré-operatório. As principais inadequações identificadas foram: período de prescrição do tratamento antimicrobiano (63,5\%); escolha do esquema de antimicrobianos (59,3\%) e dose dos antimicrobianos (58,0\%). Apenas $14,1 \%$ dos pacientes foram imunizados contra o tétano.

Conclusão As maiores divergências com o Protocolo Clínico foram observadas nas questões envolvendo o esquema de antimicrobianos utilizados, doses e tempo de prescrição, bem como na profilaxia antitetânica.

Objective To evaluate whether the conducts involving antimicrobial treatment and prophylaxis against tetanus have been performed according to the Clinical Protocol of the Institution.

Methods Descriptive and retrospective study conducted in patients of both genders, $>18$ years old admitted to a public hospital specialized in emergency and trauma, to treat primary open fracture. The data of interest were surveyed in medical records,

Trabalho feito na Fundação Hospitalar de Minas Gerais (Fhemig), Hospital João XXIII, Belo Horizonte, Minas Gerais, Brasil.

recebido

30 de Abril de 2018

aceito

28 de Março de 2019
DOI https://doi.org/

$10.1055 / \mathrm{s}-0039-3402470$. ISSN 0102-3616.
Copyright $\odot 2020$ by Sociedade Brasileira License terms de Ortopedia e Traumatologia. Published by Thieme Revinter Publicações Ltda, Rio de Janeiro, Brazil 
drug prescriptions, report of patients admitted in the Surgical Block and tetanus prophylaxis requests.

Results A total of 241 patients were selected, mostly male (81.7\%), young adults (64.3\%), victims of motorcycle accidents (53.5\%). Infectious complications were present in $18.7 \%$ of the fractures, the mean time for the surgical approach was 4 hours and 12 minutes, and $91.7 \%$ of the patients had preoperative antimicrobial prescription. The main inadequacies identified were: period of prescription of antimicrobial treat-

Keywords

- open fracture

- antibiotic prophylaxis

- infection

- tetanus ment (63.5\%); choice of the antimicrobial scheme (59.3\%) and antimicrobial dose (58.0\%). Only $14.1 \%$ of the patients were immunized against tetanus.

Conclusion The greatest divergences with the Clinical Protocol were observed in the issues involving the antimicrobial regimen used, doses and time of prescription, as well as in tetanus prophylaxis.

\section{Introdução}

As fraturas expostas (FEs) são aquelas que apresentam comunicação do foco fratuário com o meio externo ou cavidades contaminadas, por meio de uma lesão de partes moles, o que favorece a contaminação e dificulta a consolidação. Consequentemente, são fraturas de difícil abordagem clínica e pior prognóstico. ${ }^{1}$ Em grandes centros urbanos, a causa mais comum das FEs são os acidentes de trânsito, com acometimento de indivíduos do sexo masculino, em idade economicamente ativa. ${ }^{2,3}$

Existem diversas classificações para FEs, com o objetivo de escalonar a gravidade das lesões e o grau de contaminação, o que repercute no prognóstico e na escolha do tratamento. ${ }^{1}$ No sistema proposto por Gustilo et al, ${ }^{4}$ que leva em consideração a energia do trauma, o grau de lesão de partes moles e o grau de contaminação, as FEs são classificadas em tipo I, II e III. ${ }^{1,5}$ Quanto maior o nível da classificação, maior a extensão, gravidade, acometimento de partes moles, contaminação e, consequentemente, maior risco de infecções. ${ }^{6}$

Visando minimizar a incidência de complicações infecciosas, o tratamento antimicrobiano é preconizado de preferência iniciando nas primeiras horas do atendimento da $\mathrm{FE}^{5,7} \mathrm{~A}$ administração endovenosa de antimicrobiano tem papel protetor contra o desenvolvimento de infecção ${ }^{8}$ e, quanto mais precoce for iniciada, melhor o resultado. ${ }^{9}$

Outra conduta que deve ser observada no tratamento primário da FE é a profilaxia do tétano, ${ }^{5,9}$ que é uma doença infecciosa, potencialmente fatal, porém prevenível através da imunização. A transmissão ocorre pela introdução do bacilo Clostridium tetani em ferimentos geralmente perfurantes, contaminados com terra, poeira, fezes de animais ou humanas. Clinicamente, a doença se manifesta com sintomas neurotóxicos, decorrentes da ação da toxina produzida pelo bacilo. ${ }^{10}$

Na Fundação Hospitalar do Estado de Minas Gerais (FHEMIG), as diretrizes para atendimento inicial dos pacientes com FE são estabelecidas pelo Protocolo Clínico (PC) "Tratamento Primário das Fraturas Expostas". Dentre os objetivos deste Protocolo, está a redução das infecções. ${ }^{11}$ Para isso, foram padronizadas diversas condutas, dentre elas as envolvendo o tratamento inicial das fraturas expostas com antimicrobianos e a profilaxia contra 0 tétano. Tanto o tratamento antimicrobiano como a profilaxia antitetânica devem ser empregadas de maneira racional, objetivando além de garantir a eficácia e segurança no uso dos antimicrobianos e produtos imunobiológicos, melhor gerenciar os recursos utilizados na assistência ao paciente. ${ }^{12}$

No entanto, para uma assistência adequada, a mera elaboração e publicação de protocolos não são suficientes. Também são necessárias estratégias para avaliar se as condutas acordadas estão sendo aderidas. Uma dessas estratégias é a Auditoria Clínica (AC), processo estruturado na avaliação da prática clínica segundo normas estabelecidas, acompanhado de medidas educativas e implementação de mudanças, de acordo com as fragilidades encontradas. ${ }^{13}$

Assim, o presente estudo, na forma de AC, tem como objetivo avaliar se as condutas envolvendo o tratamento com antimicrobianos e a profilaxia antitetânica no atendimento inicial de pacientes com fratura exposta têm sido realizadas de acordo com as diretrizes estabelecidas pelo PC adotado na instituição.

\section{Métodos}

A AC foi realizada por meio de um estudo descritivo e retrospectivo, no qual foram analisados os atendimentos iniciais para tratamento cirúrgico de $\mathrm{FE}$, realizados no período de junho a dezembro de 2016, em hospital público estadual especializado em urgência e trauma. Foram selecionados pacientes de ambos os gêneros, $>18$ anos, com fratura do esqueleto apendicular, exceto fraturas na região da mão, uma vez que seguem protocolo próprio.

Os dados foram pesquisados nos prontuários médicos, prescrições de medicamentos do Sistema de Gestão Hospitalar (SIGH), relatórios de pacientes admitidos no Bloco Cirúrgico para cirurgia de urgência de FE e solicitações de profilaxia antitetânica.

Os pacientes foram caracterizados segundo gênero, idade e procedência. As FEs foram avaliadas de acordo com o mecanismo do trauma, segmento acometido e classificação de Gustilo. Osteomielite e infecção de partes moles foram as complicações infecciosas pesquisadas, sendo estas identificadas através de diagnóstico médico conclusivo registrado no prontuário do paciente por médicos da equipe de traumatologia.

Para avaliação do tratamento inicial com antimicrobiano (s); o esquema de antimicrobianos utilizados, doses, período 
de utilização prescrito, tempo decorrido entre a admissão e o início do tratamento antimicrobiano e o tempo de espera para a cirurgia foram confrontados com as recomendações do PC, sendo essas detalhadas no - Quadro 1.

A indicação da profilaxia antitetânica foi avaliada de acordo com o histórico vacinal do paciente registrado no prontuário. Também foi investigado o período de internação para abordagem inicial e tratamento da FE, além do fluxo de atendimento aos pacientes dentro da rede hospitalar.

Na análise estatística, foram obtidas frequências, medidas de tendência central e de dispersão. Para avaliar a associação entre as variáveis categóricas foi utilizado o teste qui quadrado de Pearson $\left(X^{2}\right)$, com nível de significância a $5 \%$ $(p<0,05)$. O estudo foi aprovado no Comitê de Ética em pesquisa da instituição sediadora, protocolo 2.211.687/2017

\section{Resultados}

No período estudado, foram identificados 241 pacientes com FE, predominantemente adultos jovens (64,3\%), homens

Quadro 1 Recomendações relacionadas ao tratamento antimicrobiano e profilaxia antitetânica do Protocolo

“Tratamento Primário das Fraturas Expostas” - FHEMIG, 2014.

\begin{tabular}{|c|c|}
\hline ANTIMICROBIANOS & RECOMENDAÇÕES \\
\hline $\begin{array}{l}\text { Tempo para } \\
\text { o início }\end{array}$ & $\begin{array}{l}\text { Recomenda-se que seja iniciada no } \\
\text { período pré- operatório }\end{array}$ \\
\hline \multirow[t]{5}{*}{$\begin{array}{l}\text { Escolha do } \\
\text { antimicrobiano }\end{array}$} & $\begin{array}{l}\text { Fraturas do tipo I: cefazolina } 1 \mathrm{~g} \\
\text { EV de } 6 / 6 \text { horas }\end{array}$ \\
\hline & $\begin{array}{l}\text { Fraturas do tipo II: cefazolina } 1 \mathrm{~g} \\
\text { EV de } 6 / 6 \text { horas + gentamicina } \\
240 \mathrm{mg} \text { EV de } 24 / 24 \text { horas }\end{array}$ \\
\hline & $\begin{array}{l}\text { Esquema alternativo para fraturas } \\
\text { dos tipos I e II: clindamicina } \\
600 \mathrm{mg} \text { EV de } 6 / 6 \text { horas }+ \\
\text { Gentamicina } 240 \mathrm{mg} \text { EV de } \\
24 / 24 \text { horas }\end{array}$ \\
\hline & $\begin{array}{l}\text { Fraturas do tipo III: cefazolina } 1 \mathrm{~g} \\
\text { EV de } 6 / 6 \text { horas + gentamicina } \\
240 \mathrm{mg} \text { EV de } 24 / 24 \text { horas }+ \\
\text { metronidazol } 500 \mathrm{mg} \text { de } 6 / 6 \text { horas }\end{array}$ \\
\hline & $\begin{array}{l}\text { Fraturas que ocorreram em } \\
\text { ambiente rural e nas fraturas } \\
\text { com sujeira grosseira deve-se } \\
\text { adicionar metronidazol } \\
500 \text { mg de } 6 / 6 \text { horas } \\
\text { aos esquemas. }\end{array}$ \\
\hline \multirow{2}{*}{$\begin{array}{l}\text { Tempo } \\
\text { de uso }\end{array}$} & Tipo I e II: 24 horas \\
\hline & Tipo III : 72 horas \\
\hline \multirow{3}{*}{$\begin{array}{l}\text { PROFILAXIA } \\
\text { DO TÉTANO }\end{array}$} & RECOMENDAÇÕES \\
\hline & $\begin{array}{l}\text { Avaliar a necessidade de acordo } \\
\text { com o histórico vacinal do paciente. }\end{array}$ \\
\hline & $\begin{array}{l}\text { Nos casos duvidosos de profilaxia } \\
\text { antitetânica prévia: deve-se } \\
\text { adicionar metronidazol } 500 \mathrm{mg} \\
\text { de } 6 / 6 \text { horas aos esquemas. }\end{array}$ \\
\hline
\end{tabular}

Fonte: Protocolo Clinico “Tratamento Primário das Fraturas Expostas” - FHEMIG,2014 (adaptado).
(81,7\%), vítimas de acidentes motociclisticos (53,5\%). O segmento anatômico mais acometido foram os membros inferiores (82,6\%) ( - Tabela 1).

Quanto à classificação de Gustilo, foram identificadas 48 (20,0\%) fraturas do tipo I, 46 (19,0\%) do tipo II e 52 (21,6\%) do tipo III. A classificação não foi registrada em prontuário em 39,4\% das FEs. Complicações infecciosas, após a abordagem inicial, ocorreram em 18,7\% das FEs, principalmente nas fraturas do tipo III (36,5\%).

O tempo médio de espera para a cirurgia de urgência foi de 4 horas e 12 minutos \pm 3 horas e 18 minutos, variando

Tabela 1 Caracterização por gênero, faixa etária, procedência, mecanismo do trauma e segmento anatômico acometido de pacientes que receberam tratamento primário de fraturas expostas, período de junho a dezembro de 2016, Hospital João XXIII, Belo Horizonte, MG.

\begin{tabular}{|c|c|c|}
\hline \multirow[t]{3}{*}{ Variáveis } & \multicolumn{2}{|c|}{$\begin{array}{l}\text { Pacientes que } \\
\text { receberam } \\
\text { tratamento } \\
\text { primário de fratura } \\
\text { exposta }\end{array}$} \\
\hline & \multicolumn{2}{|c|}{$(n=241)$} \\
\hline & $n$ & $(\%)$ \\
\hline \multicolumn{3}{|l|}{ Gênero } \\
\hline Homens & 197 & 81,7 \\
\hline Mulheres & 44 & 18,3 \\
\hline \multicolumn{3}{|c|}{ Faixa etária $(37,2 \pm 16,0$ anos; $18-86$ anos $)$} \\
\hline 18-39 anos & 155 & 64,3 \\
\hline $40-59$ anos & 62 & 25,7 \\
\hline$\geq 60$ anos & 24 & 10,0 \\
\hline \multicolumn{3}{|l|}{ Procedência } \\
\hline $\mathrm{RMBH}$ & 192 & 79,7 \\
\hline Interior do Estado de MG & 48 & 19,9 \\
\hline Não informado & 1 & 0,4 \\
\hline \multicolumn{3}{|l|}{ Mecanismo do trauma } \\
\hline Acidente automobilístico & 15 & 6,2 \\
\hline Acidente de bicicleta & 5 & 2,1 \\
\hline Acidentes com máquinas & 10 & 4,1 \\
\hline Acidente motociclístico & 129 & 53,5 \\
\hline Agressão física & 5 & 2,1 \\
\hline Atropelamento & 33 & 13,7 \\
\hline $\begin{array}{l}\text { Agressão por arma } \\
\text { de fogo ou branca }\end{array}$ & 8 & 3,3 \\
\hline Queda & 32 & 13,3 \\
\hline Outros & 4 & 1,7 \\
\hline \multicolumn{3}{|c|}{ Segmento anatômico acometido } \\
\hline Membro inferior & 199 & 82,6 \\
\hline Membro superior & 38 & 15,8 \\
\hline $\begin{array}{l}\text { Membro inferior }+ \\
\text { membro superior }\end{array}$ & 4 & 1,6 \\
\hline
\end{tabular}

Abreviações: MG, Minas Gerais; RMBH, região metropolitana de Belo Horizonte.

Fonte: os autores. 
entre 38 minutos até 21 horas e 7 minutos, sendo que 199 $(82,6 \%)$ pacientes foram operados até 6 horas após a admissão. O tratamento antimicrobiano foi prescrito no pré-operatório para $221(91,7 \%)$ pacientes, e $172(71,7 \%)$ tiveram prescrição do tratamento com antimicrobianos em até 3 horas após a admissão. Houve associação estatisticamente significativa entre complicação infecciosa e fratura do tipo III $(p=0,0014)$, tratamento antimicrobiano iniciado no período pós-operatório $(p=0,0362)$ e tratamento anti- microbiano iniciado após 3 horas da admissão do paciente $(p=0,0350)$, dados estes apresentados na - Tabela 2.

Do total de 241 pacientes, 74 (30,7\%) foram excluídos da avaliação do tratamento antimicrobiano por falta de registro da classificação da fratura e inexistência de critério para uso do tratamento antimicrobiano para fratura tipo III, nos casos de trauma há mais de 6 horas.

As inadequações relativas à escolha do esquema antimicrobiano, doses prescritas e a duração do tratamento

Tabela 2 Distribuição das complicações infecciosas por classificação da fratura, tempo de espera para a abordagem cirúrgica e tempo decorrido entre a admissão e o inicio do tratamento antimicrobiano, período de junho a dezembro de 2016, Hospital João XXIII, Belo Horizonte, MG.

\begin{tabular}{|c|c|c|c|c|c|c|c|}
\hline \multirow[t]{3}{*}{ Variáveis } & \multicolumn{2}{|c|}{$\begin{array}{l}\text { Pacientes com } \\
\text { fratura } \\
\text { exposta ( } n \\
=241 \text { ) } \\
\end{array}$} & \multicolumn{4}{|c|}{$\begin{array}{l}\text { Complicações } \\
\text { infecciosas }\end{array}$} & \multirow[t]{3}{*}{ valor-p* } \\
\hline & \multirow[b]{2}{*}{$n$} & \multirow[b]{2}{*}{$(\%)$} & \multicolumn{2}{|c|}{$\operatorname{SIM}(n=45)$} & \multicolumn{2}{|c|}{ NÃO $(n=196)$} & \\
\hline & & & $n$ & (\%) & $n$ & (\%) & \\
\hline \multicolumn{8}{|l|}{ Classificação da FE } \\
\hline Tipo I & 048 & $(20,0)$ & 05 & $(10,4)$ & 043 & $(89,6)$ & $p=0,0014$ \\
\hline Tipo II & 046 & $(19,0)$ & 04 & $(08,7)$ & 042 & $(91,3)$ & \\
\hline Tipo III & 052 & $(21,6)$ & 19 & $(36,5)$ & 033 & $(63,5)$ & \\
\hline Não classificada & 095 & $(39,4)$ & 17 & $(17,9)$ & 078 & $(82,1)$ & \\
\hline \multicolumn{8}{|c|}{$\begin{array}{l}\text { Tempo decorrido entre a admissão do paciente } \\
\text { e a abordagem cirúrgica }\end{array}$} \\
\hline$\leq 6$ horas & 199 & $(82,6)$ & 37 & $(18,6)$ & 162 & $(81,4)$ & $p=0,4621$ \\
\hline$>6$ horas & 042 & $(17,4)$ & 08 & $(19,0)$ & 034 & $(81,0)$ & \\
\hline \multicolumn{8}{|c|}{$\begin{array}{l}\text { Tempo decorrido entre a admissão do paciente } \\
\text { e o inicio do tratamento antimicrobiano }\end{array}$} \\
\hline Pré-operatório & 221 & $(91,7)$ & 38 & $(17,2)$ & 183 & $(82,8)$ & $p=0,0362$ \\
\hline Pós-operatório & 020 & $(08,3)$ & 07 & $(35,0)$ & 013 & $(65,0)$ & \\
\hline$\leq 3$ horas após admissão & 172 & $(71,7)$ & 27 & $(15,7)$ & 145 & $(84,3)$ & $p=0,0350$ \\
\hline$>3$ horas após admissão & 069 & $(28,3)$ & 18 & $(26,1)$ & 051 & $(73,9)$ & \\
\hline TOTAL & 241 & $(100)$ & 45 & $(18,7)$ & 196 & $(81,3)$ & \\
\hline
\end{tabular}

Legenda: ${ }^{*}$ Teste $x^{2}$ - Nível de significância: $p<0,05 *$ Teste $x^{2}$.

Fonte: os autores.

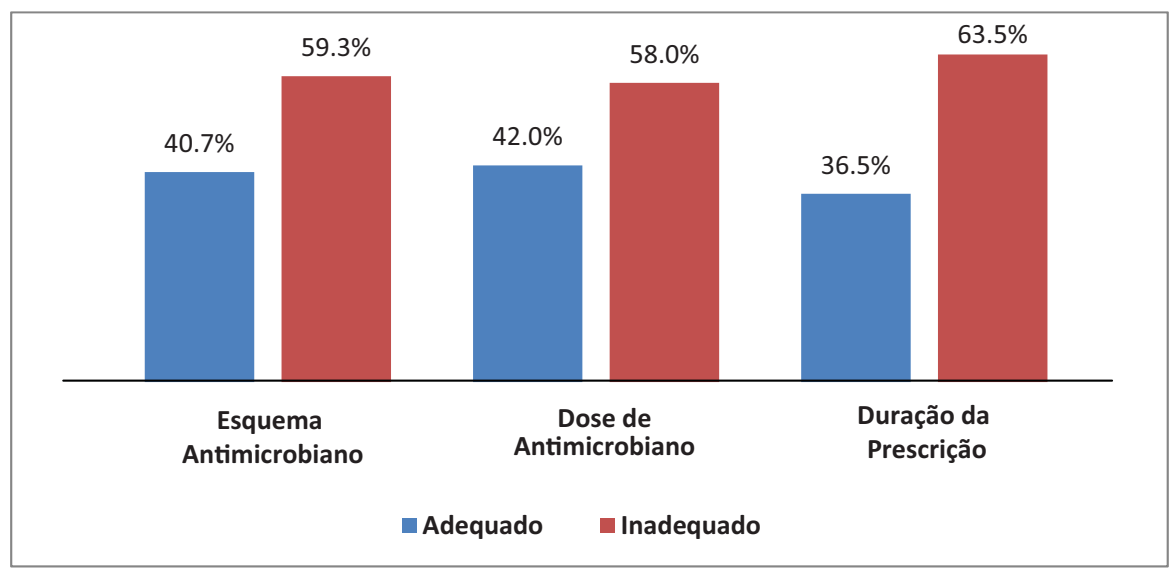

Fig. 1 Distribuição das adequações e inadequações dos esquemas de antimicrobianos, doses prescritas e duração da prescrição, de acordo com as diretrizes do Protocolo Clínico “Tratamento Primário das Fraturas Expostas" - FHEMIG, período de junho a dezembro de 2016, Hospital joão XXIII, Belo Horizonte - MG. Fonte: os autores. 
antimicrobiano superaram as adequações, conforme representado na - Figura 1.

Em relação aos agentes antimicrobianos, 59,3\% dos pacientes foram expostos a associações não recomendadas no PC. A maior porcentagem de inadequações foi observada nos portadores de FE do tipo III e/ou trauma com ocorrência há mais de 6 horas $(75,0 \%)$ e nos portadores de FE do tipo II (40,0\%). As principais inadequações foram, respectivamente, ausência da prescrição médica do metronidazol para cobertura contra bactérias anaeróbicas (55,5\%) e a ausência da prescrição médica da gentamicina para cobertura ampliada contra Gram negativos $(87,4 \%)$ ( - Tabela 3 ).
Considerando o total de 438 antimicrobianos prescritos para os esquemas de tratamentos avaliados, 254 (58,0\%) apresentaram doses diárias divergentes do PC. A maior parte das inadequações ocorreu nas prescrições de cefazolina $(93,0 \%)$ e de clindamicina (86,0\%). Ao contrário, a maioria das doses prescritas de metronidazol $(65,0 \%)$ estavam adequadas. Quanto à gentamicina, todas as doses observadas estavam de acordo com o PC (-Figura 2).

Quanto à duração, 63,5\% dos tratamentos com antimicrobianos permaneceram prescritos por tempo inadequado. Os resultados mais expressivos foram observados nos portadores de FE de tipos I e II $(95,6 \%)$, que foram expostos ao

Tabela 3 Descrição dos tipos de inadequações, por tipo de fratura, envolvendo esquemas de antimicrobianos, de acordo com o protocolo Tratamento Primário das Fraturas Expostas - FHEMIG, período de junho a dezembro de 2016, Hospital João XXIII, Belo Horizonte, MG.

\begin{tabular}{|c|c|c|}
\hline \multirow[t]{2}{*}{ TRATAMENTO ANTIMICROBIANO } & \multicolumn{2}{|c|}{ Pacientes $(n=167)$} \\
\hline & $n$ & $(\%)$ \\
\hline \multicolumn{3}{|l|}{ Tipo I } \\
\hline Adequada & 20 & $(64,5)$ \\
\hline Inadequada & 11 & $(35,5)$ \\
\hline $\begin{array}{l}\text { Trauma ocorrido em ambiente rural } \\
\text { e/ou contaminação com sujidades } \\
\text { sem cobertura para anaeróbicos }\end{array}$ & 03 & $(27,3)$ \\
\hline $\begin{array}{l}\text { Cobertura ampliada para Gram (-) } \\
\text { desnecessária }\end{array}$ & 03 & $(27,3)$ \\
\hline $\begin{array}{l}\text { Cobertura ampliada para Gram (-) } \\
\text { e anaeróbicos desnecessária }\end{array}$ & 04 & $(36,3)$ \\
\hline $\begin{array}{l}\text { Esquema alternativo: Clindamicina } \\
\text { não associada à gentamicina }\end{array}$ & 01 & $(09,1)$ \\
\hline \multicolumn{3}{|l|}{ Tipo II } \\
\hline Adequada & 24 & $(60,0)$ \\
\hline Inadequada & 16 & $(40,0)$ \\
\hline $\begin{array}{l}\text { Trauma ocorrido em ambiente } \\
\text { rural e/ou contaminação com } \\
\text { sujidades sem cobertura para } \\
\text { anaeróbico }\end{array}$ & 01 & $(06,3)$ \\
\hline Sem cobertura ampliada para Gram (-) & 14 & $(87,4)$ \\
\hline $\begin{array}{l}\text { Cobertura desnecessária para } \\
\text { anaeróbicos }\end{array}$ & 01 & $(06,3)$ \\
\hline \multicolumn{3}{|l|}{ Tipo III e/ou trauma há mais de 6 horas } \\
\hline Adequada & 24 & $(25,0)$ \\
\hline Inadequada & 72 & $(75,0)$ \\
\hline Sem cobertura para anaeróbicos & 40 & $(55,5)$ \\
\hline $\begin{array}{l}\text { Sem cobertura ampliada para } \\
\text { Gram (-) e anaeróbicos }\end{array}$ & 21 & $(29,2)$ \\
\hline Sem cobertura ampliada para Gram (-) & 06 & $(08,3)$ \\
\hline $\begin{array}{l}\text { Uso de antimicrobiano não } \\
\text { recomendado (ceftriaxona) }\end{array}$ & 05 & $(06,9)$ \\
\hline Adequações & 68 & $(040,7)$ \\
\hline Inadequações & 99 & $(059,3)$ \\
\hline
\end{tabular}

Fonte: os autores. 


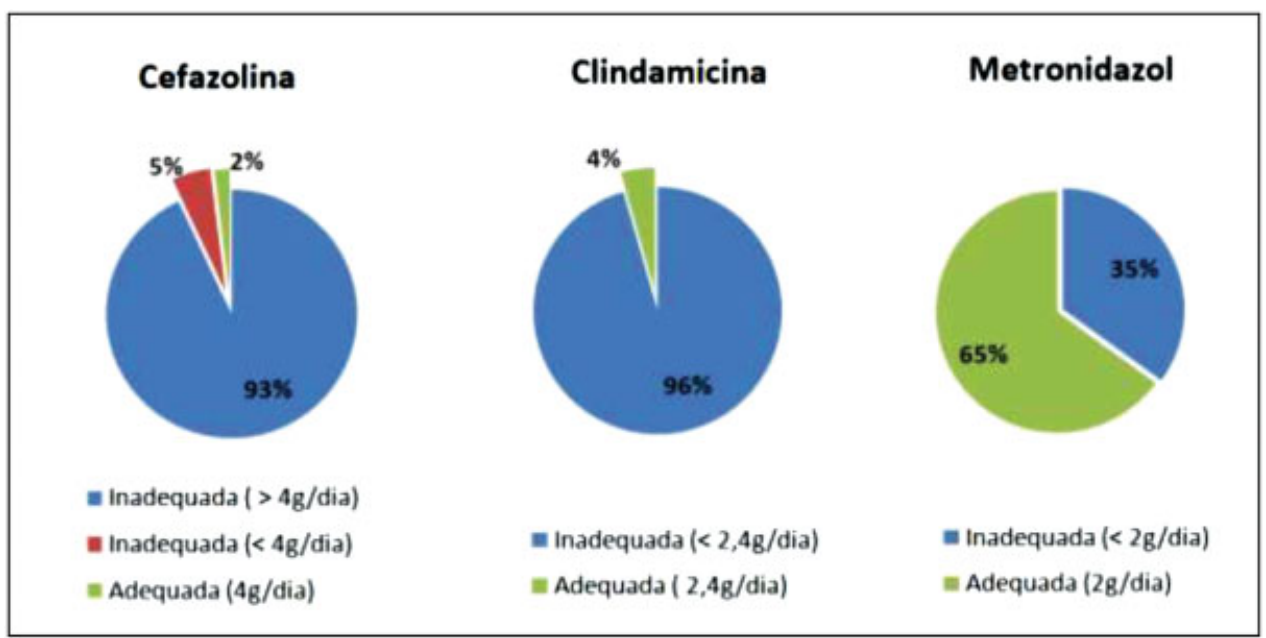

Fig. 2 Análise das adequações e inadequações relacionadas às doses dos antimicrobianos prescritos, de acordo com o Protocolo "Tratamento Primário das Fraturas Expostas" - FHEMIG, período de junho a dezembro de 2016, Hospital João XXIII, Belo Horizonte - MG. Fonte: os autores.

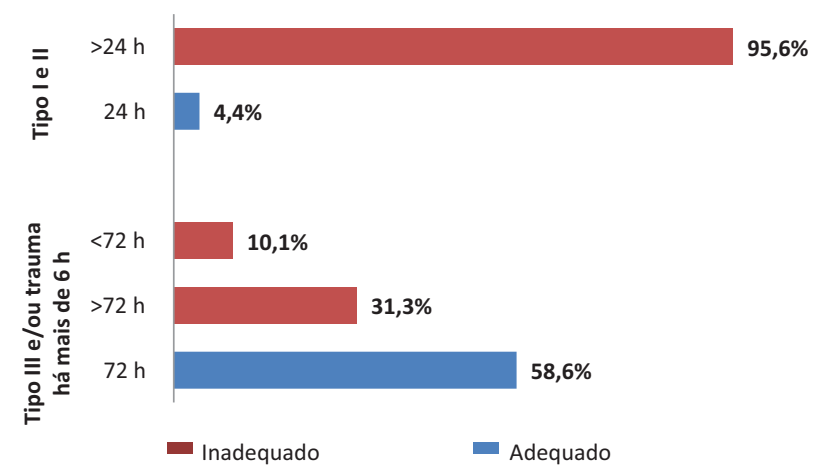

Fig. 3 Análise das adequações e inadequações relacionadas ao tempo de prescrição do tratamento com antimicrobianos, de acordo com as diretrizes do Protocolo “Tratamento Primário das Fraturas Expostas”, período de junho a dezembro de 2016, Hospital João XXIII, Belo Horizonte - MG. Fonte: os autores. tratamento antimicrobiano por período superior a 24 horas (-Figura 3).

O período médio de prescrição de antimicrobianos nos portadores de FE tipos I e II foi de 4 dias, com variação entre 1 a 10 dias, enquanto que no PC a indicação seria de apenas 1 dia. Já nos portadores de FE do tipo III e/ou trauma com ocorrência há mais de 6 horas, o período médio de prescrição obtido foi de 3 dias, com variação entre 1 a 9 dias, sendo que $o$ tempo indicado no PC seria de 3 dias. Em termos globais, observou-se que a média de duração da prescrição de antimicrobianos foi maior nos pacientes transferidos para outras unidades da rede hospitalar (4 dias, com variação entre 1 a 10 dias) do que nos pacientes que permaneceram no hospital de origem ( 3 dias, com variação entre 1 a 8 dias).

Com relação à profilaxia antitetânica, não foram localizados nos prontuários os registros do histórico vacinal de 207 (85,9\%) portadores de FE, bem como não houve prescrição da

Tabela 4 Tempo de permanência dos pacientes com fratura exposta, por tipo de fratura e complicação infecciosa, no hospital que realizou o atendimento inicial e após a transferência para outra unidade da rede hospitalar

\begin{tabular}{|c|c|c|c|c|c|c|c|c|c|}
\hline \multirow[t]{3}{*}{ Variáveis } & \multicolumn{9}{|c|}{$\begin{array}{l}\text { Tempo de Permanência } \\
\text { (Dias) }\end{array}$} \\
\hline & \multicolumn{3}{|c|}{$\begin{array}{l}\text { Hospital que Realizou o } \\
\text { Tratamento Inicial }\end{array}$} & \multicolumn{3}{|c|}{$\begin{array}{l}\text { Outra Unidade da Rede } \\
\text { Hospitalar }\end{array}$} & \multicolumn{3}{|c|}{ Total na Rede Hospitalar } \\
\hline & Média & MD & AMP & Média & MD & AMP & Média & MD & AMP \\
\hline \multicolumn{10}{|c|}{ Classificação da fratura } \\
\hline Tipo I & 10 & 04 & $1-35$ & 11 & 08 & $1-41$ & 15 & 09 & $1-137$ \\
\hline Tipo II & 05 & 02 & $1-42$ & 09 & 05 & $1-48$ & 10 & 06 & $1-60$ \\
\hline Tipo III & 17 & 09 & $1-93$ & 14 & 13 & $1-72$ & 25 & 19 & $1-107$ \\
\hline Sem classificação & 07 & 04 & $1-79$ & 09 & 07 & $1-50$ & 13 & 08 & $1-77$ \\
\hline \multicolumn{10}{|c|}{ Complicação infecciosa } \\
\hline Sem infecção & 07 & 04 & $1-079$ & 07 & 04 & $1-41$ & 12 & 07 & $1-77$ \\
\hline Com infecção & 21 & 15 & $1-135$ & 16 & 12 & $1-72$ & 37 & 30 & $4-137$ \\
\hline Total & 09 & 04 & $1-135$ & 11 & 8 & $1-72$ & 16 & 09 & $1-137$ \\
\hline
\end{tabular}

Abreviações: AMP, amplitude; MD, mediana.

Fonte: os autores. 
imunização para esses pacientes. Somente 34 (14,1\%) pacientes foram imunizados, sendo que $28(82,3 \%)$ estavam internados na Unidade de Terapia Intensiva (UTI).

Quanto ao fluxo de atendimento aos pacientes, 124 $(51,4 \%)$ portadores de FE foram transferidos para outras unidades da rede hospitalar, 91 (37,8\%) completaram o tratamento no hospital que realizou o atendimento inicial, $21(8,7 \%)$ foram transferidos para hospitais da rede privada e $5(2,1 \%)$ foram a óbito. O tempo médio de permanência na rede hospitalar foi de 16 dias, sendo maior nos portadores de FE do tipo III (25 dias) e naqueles que tiveram complicações infecciosas (37 dias), conforme a -Tabela 4.

\section{Discussão}

Com o desenvolvimento tecnológico e diante da diversidade de opções diagnósticas e terapêuticas existentes, os PC surgiram para reduzir a variabilidade de condutas adotadas e auxiliar os profissionais da área da saúde na tomada de decisão, de forma a garantir a qualidade e segurança da assistência prestada ao paciente. São elaborados após um amplo estudo das melhores evidências científicas e consensos disponíveis na literatura sobre determinado assunto. ${ }^{14}$

O PC que norteou a AC padronizou o tratamento com antimicrobianos no primeiro atendimento de pacientes com $\mathrm{FE}$, de acordo com a classificação da fratura, o que auxilia na escolha das melhores condutas e na previsão do prognóstico. Apesar disso, o estudo evidenciou uma expressiva frequência de fraturas sem registro de classificação no prontuário. Tal inadequação inviabilizou a avaliação do uso de antimicrobianos nessas fraturas, exceto naquelas com trauma ocorrido há mais de 6 horas, pois, neste caso, o esquema recomendado é o mesmo utilizado para pacientes com fratura do tipo III.

O perfil epidemiológico dos participantes do estudo foi semelhante ao encontrado na literatura, com maior acometimento de jovens do sexo masculino. ${ }^{15-18}$ Esse fato pode ser explicado pela maior exposição dessa população aos acidentes de trânsito, especialmente os motociclísticos.

A taxa de infecção encontrada $(18,7 \%)$ foi condizente com o resultado de um estudo realizado em hospital de prontoatendimento localizado em Canoas, RS (18,8\%). ${ }^{18}$ Entretanto, outros estudos, sendo um nacional ${ }^{16}$ e um internacional, ${ }^{19}$ encontraram taxas menores, de 10,0\% e 13,2\%, respectivamente. A significativa associação entre complicações infecciosas e fratura do tipo III encontrada na AC é uma relação já bem estabelecida na literatura. ${ }^{18,19}$

A AC também evidenciou que o tempo médio de espera para cirurgia de urgência foi de 4 horas e 12 minutos, resultado este abaixo do limite de 6 horas recomendado no PC. Um estudo realizado no Canadá encontrou tempo médio de espera de 9 horas e 15 minutos. ${ }^{17}$ Assim como em outras publicações, na AC não foi demonstrada associação entre o tempo de espera para a cirurgia de urgência e a presença de complicações infecciosas. $^{16,17}$

A associação positiva entre menor frequência de complicações infecciosas e tempo entre a admissão e o início do tratamento antimicrobiano encontrada na auditoria também foi observada no estudo descritivo de Lack et al. ${ }^{20}$ Embora o PC recomende que o inicio do uso de antimicrobianos deva ser instituído no período pré-operatório, sem estabelecer o limite de tempo entre a admissão e o início do tratamento, estudos demonstraram que o atraso na administração de antimicrobianos além de 3 horas está relacionado ao maior risco de infecções. ${ }^{21}$

Foi considerado preocupante o fato das maiores taxas de inadequação do emprego de antimicrobianos ter ocorrido nas FEs dos tipos II e III, uma vez que essas fraturas são as que têm maior propensão de complicações infecciosas. ${ }^{22}$

No PC, a posologia dos antimicrobianos foi definida levando-se em conta o perfil epidemiológico das FEs no cenário de estudo, para indivíduos com peso médio de 70 $\mathrm{Kg}$, considerando-se predominância de adultos jovens, previamente hígidos. Esta padronização visa facilitar o manejo desses agentes em situações de emergência, além de garantir o uso racional dos antimicrobianos, evitando-se doses, frequências e/ou períodos de uso inadequados. Porém, o PC não prevê situações nas quais outros perfis de indivíduos são acometidos de FE, como obesos, quando a dose de antimicrobiano deve ser individualizada e calculada de acordo com o peso corporal. Por este motivo, doses de antimicrobianos não padronizados no PC foram consideradas inadequadas, embora estejam na faixa terapêutica estabelecida pela literatura, quando se leva em conta o peso corporal do paciente. Desse modo, é importante que esses casos sejam previstos no PC, flexibilizando-se as doses dos antimicrobianos de acordo com as características individuais dos pacientes. ${ }^{23,24}$

O período prolongado de prescrição dos antimicrobianos foi um dos resultados que mais chamou atenção, principalmente nas FEs dos tipos I e II, com utilização por tempo médio quatro vezes superior à recomendação do PC. Cabe também ressaltar que a maior duração do tratamento antimicrobiano ocorreu na transição do cuidado para outras unidades da rede hospitalar, provavelmente por falha na consulta ao prontuário sobre o tempo de uso do antimicrobiano pelo paciente no hospital de origem. Apesar das controvérsias entre diversos autores sobre a duração apropriada do tratamento antimicrobiano, estudos atuais demonstram que o aumento do tempo de exposição não tem influência na redução das taxas de complicações infecciosas. ${ }^{25,26}$ Um estudo retrospectivo caso controle que comparou a taxa de infecção entre pacientes com FE submetidos ao uso de antimicrobianos por períodos que variaram de $1 \mathrm{a}>5$ dias não indicou diferenças significativas no risco de infecção, inclusive nas FEs do tipo III. ${ }^{25}$ Além de não trazer benefícios, a duração prolongada do tratamento antimicrobiano expõe os pacientes ao maior risco de eventos adversos, desenvolvimento de resistência bacteriana, aumento do tempo de permanência hospitalar e elevação dos custos relacionados à assistência. ${ }^{5,6}$

Com relação à profilaxia antitetânica, o PC somente menciona que a profilaxia deve ser realizada, sem detalhar as condutas, que devem ser baseadas nas diretrizes nacionais. ${ }^{11}$ Recomenda apenas associação de metronidazol para pacientes com histórico de vacinação desconhecido ou incerto, devido à atividade antianaeróbica deste agente, o que reduz a carga bacteriana no foco da inoculação e evita a 
produção da toxina do tétano. ${ }^{27}$ No entanto, a utilização do metronidazol não exclui a necessidade de imunoprofilaxia. Entretanto, no período auditado, não havia registro do histórico vacinal para a totalidade dos portadores de FE, o que foi considerado uma grave inadequação, tendo em vista as prováveis oportunidades perdidas de vacinação. Desse modo, na presença de FE, é necessario averiguar e registrar em prontuário o histórico vacinal do paciente, para adoção das estratégias adequadas.

A atuação do farmacêutico clínico na UTI, na revisão de cuidados básicos aplicáveis a pacientes críticos, o que inclui a investigação do estado vacinal, talvez possa explicar que a maior parte dos pacientes com FE imunizados estavam internados nesta unidade. Resultados positivos com a participação do farmacêutico na equipe de tratamento do trauma já foram relatados em outros trabalhos, o que reforça a importância desse profissional como membro da equipe multidisciplinar de cuidado ao paciente. ${ }^{28}$

Como limitação do presente estudo, é importante comentar o fato de que a pesquisa objetivou avaliar somente o cumprimento do PC pela equipe de traumatologia, quanto ao primeiro atendimento dos pacientes com FE, não sendo consideradas as demais comorbidades ou lesões apresentadas por esses pacientes. Tal fato pode ter influenciado nos resultados do estudo, visto que os pacientes mais graves poderiam ter a primeira abordagem ortopédica adiada para privilegiar o atendimento das condições de maior urgência, o que influenciaria em critérios avaliados no estudo, como no tempo para a primeira abordagem cirúrgica, período de internação e incidência de complicações infecciosas.

\section{Conclusão}

O presente estudo permitiu identificar as divergências em relação ao PC adotado pela instituição, sendo que as inadequações mais expressivas foram observadas nas escolhas dos esquemas de antimicrobianos utilizados, doses e tempo de tratamento, bem como na profilaxia antitetânica.

Conflito de Interesses

Os autores declaram não haver conflito de interesses.

\section{Referências}

1 Gliglio PN, Cristante AF, Pécora JR, Helito CP, Lima ALL, Silva JS. Avanços no tratamento das fraturas expostas. Rev Bras Ortop 2015;50(02):125-130

2 Müller SS, Sadenberg T, Pereira GJ, Sadatsune T, Kimura EE, Novelli Filho JL. Estudo epidemiológico, clínico e microbiológico prospectivo de pacientes portadores de fraturas expostas atendidos em hospital universitário. Acta Ortop Bras 2003;11(03): 158-169

3 Arruda LR, Silva MA, Malerba FG, Fernandes MC, Turibio FM, Matsumoto $\mathrm{MH}$. Fraturas expostas: estudo epidemiológico e prospectivo. Acta Ortop Bras 2009;17(06):326-330

4 Gustilo RB, Anderson JT. Prevention of infection in the treatment of one thousand and twenty-five open fractures of long bones: retrospective and prospective analyses. J Bone Joint Surg Am 1976;58(04):453-458
5 Rodriguez L, Jung HS, Goulet JA, Cicalo A, Machado-Aranda DA, Napolitano LM. Evidence-based protocol for prophylactic antibiotics in open fractures: improved antibiotic stewardship with no increase in infection rates. J Trauma Acute Care Surg 2014;77 (03):400-407

6 Isaac SM, Woods A, Danial IN, Mourkus H. Antibiotic prophylaxis in adults with open tibial fractures: what is the evidence for duration of administration? A systematic review. J Foot Ankle Surg 2016;55(01):146-150

7 Hauser CJ, Adams CA Jr, Eachempati SR. Council of the Surgical Infection Society. Surgical Infection Society guideline: prophylactic antibiotic use in open fractures: an evidence-based guideline. Surg Infect (Larchmt) 2006;7(04):379-405

8 Cotta AMA, Peres CF, Ribeiro DAM, et al. Antibioticoterapia e imunoprofilaxia do tétano no trauma perfurocortante. Rev Med Minas Gerais. 2009;19(02):96-103

9 Gonzalez VL, Santin E, Arsego FV, et al. Diagnóstico e manejo das lesões ortopédicas em pacientespolitraumatizados. Rev HCPA. 2009;29(02):153-160

10 Brasil. Ministério da Saúde. Guia de Vigilância Epidemiológica/Fundação Nacional de Saúde [acesso em 2018 Fev 20]. $5^{\circ}$ ed. BrasíliaFUNASA2002. Disponível em: http://bvsms.saude. gov.br/bvs/publicacoes/funasa/guia_vig_epi_vol_ll.pdf

11 Fundação Hospitalar do Estado de Minas Gerais. Diretrizes clinicas Protocolos clínicos. Tratamento primário das fraturas expostas. Revisado 2014 [acesso em 2018 Fev 20]. Disponível em: http:// www.fhemig.mg.gov.br/index.php/docman/Protocolos_Clinicos-1/ 70-019-tratamento-primario-das-fraturas-expostas-07082014/file

12 Araujo RQ. Antibióticoprofilaxia em cirurgias ortopédicas: resultado da implantação de um protocolo [tese]. São Paulo: Universidade Estadual de Campinas, Faculdade de Ciências Medicas; 2000

13 Bazzanella NA, Slob E. A auditoria como ferramenta de analise para a melhoria da qualidade do serviço prestado. Cad Saude Desenvolv. 2013;3(02):50-65

14 Mahmud SDP. Protocolos clínicos: adesão e aplicabilidade numa instituição hospitalar [tese]. Rio Grande do Sul: Universidade Federal do Rio Grande do Sul, Escola de Administração; 2002

15 Villa PEA, Nunes TR, Gonçalves FP, Martins JS, Lemos GSP, Moraes FB. Avaliação clinica de pacientes com osteomielite crônica após fraturas expostas tratadas no Hospital de Urgências de Goiânia, Goiás. Rev Bras Ortop 2013;48(01):22-28

16 Fernandes MC, Peres LR, Queiroz Neto AC, Lima Neto JQ, Turíbio FM, Matisumoto MH. Fraturas expostas e a incidência de infecções no desbridamento cirúrgico 6 horas após o trauma. Acta Ortop Bras 2015;23(01):38-42

17 Weber D, Dulai SK, Bergman J, Buckley R, Beaupre LA. Time to initial operative treatment following open fracture does not impact development of deep infection: a prospective cohort study of 736 subjects. J Orthop Trauma 2014;28(11):613-619

18 Guerra MTE, Gregio FM, Bernardi A, Castro CC. Taxa de infecção em pacientes adultos com fratura exposta atendidos no hospital de pronto socorro e no hospital universitário Ulbra do município de Canoas, Rio Grande do Sul. Rev Bras Ortop 2017;52(05): 541-548

19 Chen AF, Schreiber VM, Washington W, Rao N, Evans AR. What is the rate of methicillin-resistant Staphylococcus aureus and Gram-negative infections in open fractures? Clin Orthop Relat Res 2013;471(10):3135-3140

20 Lack WD, Karunakar MA, Angerame MR, et al. Type III open tibia fractures: immediate antibiotic prophylaxis minimizes infection. J Orthop Trauma 2015;29(01):1-6

21 Patzakis MJ, Wilkins J. Factors influencing infection rate in open fracture wounds. Clin Orthop Relat Res 1989;(243): $36-40$

22 Hoff WS, Bonadies JA, Cachecho R, Dorlac WC. East Practice Management Guidelines Work Group: update to practice management guidelines for prophylactic antibiotic use in open fractures. J Trauma 2011;70(03):751-754 
23 DynaMed Plus [database online]. Ipswich (MA): EBSCO Information Services [acesso em 2018 Feb 23]. Disponível em: http:// www.dynamed.com

24 Halawi MJ, Morwood MP. Acute management of open fractures: an evidence-based review. Orthopedics 2015;38(11):e1025e1033

25 Dunkel N, Pittet D, Tovmirzaeva L, et al. Short duration of antibiotic prophylaxis in open fractures does not enhance risk of subsequent infection. Bone Joint J 2013;95-B(06):831837
26 Messner J, Papakostidis C, Giannoudis PV, Kanakaris NK. Duration of administration of antibiotic agentes for open fractures; metaanalysis of the existing evidence. Surg Infect (Larchmt) 2017;18 (08):854-867

27 Lisboa T, Ho YL, Henriques Filho GT, et al. Diretrizes para o manejo do tétano acidental em pacientes adultos. Rev Bras Ter Intensiva 2011;23(04):394-409

28 Harvey S, Brad Hall A, Wilson K. Impact of an emergency medicine pharmacist on initial antibiotic prophylaxis for open fractures in trauma patients. Am J Emerg Med 2018;36(02):290-293 